Supporting Information

\title{
Modeling on a three-way catalyst used in series hybrid electric vehicles considering its specific engine operation attribute
}

Toshinori Okajima* a,b, Suchitra Sivakumar a , Hajime Shingyouchi a, Kyohei Yamaguchi $^{a}$, Jin Kusaka ${ }^{a}$, Makoto Nagata ${ }^{b}$.

(a Department of Modern Mechanical Engineering, Graduate School of Creative Science and Engineering, Waseda University, 3-4-1 Okubo, Shinjuku-ku, Tokyo 169-8555, Japan).

(b N.E. Chemcat Corporation, 678 Ipponmatsu, Numazu, Shizuoka 410-0314, Japan).

Corresponding author

Toshinori Okajima

E-mail: toshinori.okajima@ne-chemcat.co.jp 
Table S1 Vehicle experimental conditions.

\begin{tabular}{c|c}
\hline \hline Fuel & Regular gasoline \\
\hline Driving mode & $\begin{array}{c}\text { WLTC (except extra } \\
\text { high) }\end{array}$ \\
\hline Ambient Temperature ${ }^{\circ} \mathrm{C}$ & 23 \\
\hline Initial SOC $\%$ & 60 \\
\hline
\end{tabular}

Table S2 Major measured parameters.

\begin{tabular}{|c|c|}
\hline \multirow[t]{3}{*}{ Engine } & Speed [rpm] \\
\hline & Torque $[\mathrm{N} \cdot \mathrm{m}]$ \\
\hline & $\begin{array}{l}\left.\text { Intake pressure }[\mathrm{kPa}] \text {, temperature }{ }^{\circ} \mathrm{C}\right] \text {, } \\
\text { mass flow }[\mathrm{kg} / \mathrm{h}]\end{array}$ \\
\hline \multirow[t]{6}{*}{ Exhaust } & Gas concentration (cc-TWC In/Out) [ppm] \\
\hline & Gas Temperature (cc-TWC In/Out ) [ $\left.{ }^{\circ} \mathrm{C}\right]$ \\
\hline & $\begin{array}{l}\text { TWC Bed Temperature (Front/Mid/Rear) } \\
{\left[{ }^{\circ} \mathrm{C}\right]}\end{array}$ \\
\hline & Lambda (cc-TWC In/Out) \\
\hline & Pressure (cc-TWC In/Out) [kPa] \\
\hline & Mass Flow [kg/h] \\
\hline
\end{tabular}

Table S3 Specifications of the model gas test reactor.

\begin{tabular}{cc|c}
\hline \hline Total gas flow rate & $\mathrm{L} / \mathrm{min}$ & $5.0-75.0$ \\
\hline Maximum temperature & ${ }^{\circ} \mathrm{C}$ & (temporal) 800 \\
\hline Temperature ramping rate & ${ }^{\circ} \mathrm{C} / \mathrm{min}$ & $30-50$ \\
\hline Base test piece diameter & $\mathrm{mm}$ & 25.4 \\
\hline
\end{tabular}


Table S4 List of reactions to be provided with additional inhibition multipliers.

\begin{tabular}{|c|c|c|c|c|c|c|c|c|c|c|}
\hline \multirow{3}{*}{ \# } & \multirow{2}{*}{\multicolumn{2}{|c|}{ Reactant }} & & \multirow{2}{*}{\multicolumn{3}{|c|}{ Product }} & \multicolumn{3}{|c|}{ Inhibition Species } & \multirow{3}{*}{\begin{tabular}{|l}
$\begin{array}{l}\text { Inhibition } \\
\text { Factors }\end{array}$ \\
$G * G^{\prime}{ }_{2}$
\end{tabular}} \\
\hline & & & & & & & \multirow{2}{*}{$\frac{\mathrm{C}_{3} \mathrm{H}_{6}}{\underline{\underline{\boldsymbol{V}}}}$} & \multirow[t]{2}{*}{$\mathrm{O}_{2}$} & \multirow[t]{2}{*}{ NO } & \\
\hline & $\mathrm{C}_{3} \mathrm{H}_{8}$ & $5 \mathrm{O}_{2}$ & $\rightarrow$ & $3 \mathrm{CO}_{2}$ & $4 \mathrm{H}_{2} \mathrm{O}$ & & & & & \\
\hline 8 & $\mathrm{C}_{3} \mathrm{H}_{6}$ & $18 \mathrm{NO}$ & $\rightarrow$ & $3 \mathrm{CO}_{2}$ & $9 \mathrm{~N} 2 \mathrm{O}$ & $3 \mathrm{H}_{2} \mathrm{O}$ & & $\checkmark$ & & $G * G_{1}^{\prime}$ \\
\hline 9 & $\mathrm{C}_{3} \mathrm{H}_{6}$ & $9 \mathrm{NO}$ & $\rightarrow$ & $3 \mathrm{CO}_{2}$ & $3 \mathrm{H}_{2} \mathrm{O}$ & $4.5 \mathrm{~N}_{2}$ & & $\checkmark$ & & $G * G_{1}^{\prime}$ \\
\hline 10 & $\mathrm{C}_{3} \mathrm{H}_{8}$ & $20 \mathrm{NO}$ & $\rightarrow$ & $3 \mathrm{CO}_{2}$ & $10 \mathrm{~N}_{2} \mathrm{O}$ & $4 \mathrm{H}_{2} \mathrm{O}$ & $\checkmark$ & $\checkmark$ & & $G * G_{3}^{\prime}$ \\
\hline 11 & $\mathrm{C}_{3} \mathrm{H}_{8}$ & $10 \mathrm{NO}$ & $\rightarrow$ & $3 \mathrm{CO}_{2}$ & $4 \mathrm{H}_{2} \mathrm{O}$ & $5 \mathrm{~N}_{2}$ & $\checkmark$ & $\checkmark$ & & $G * G^{\prime}{ }_{3}$ \\
\hline 17 & $\mathrm{CO}$ & $\mathrm{H}_{2} \mathrm{O}$ & $\rightarrow$ & $\mathrm{CO}_{2}$ & $\mathrm{H}_{2}$ & & $\checkmark$ & $\checkmark$ & & $G * G_{3}^{\prime}$ \\
\hline 18 & $\mathrm{CO}_{2}$ & $\mathrm{H}_{2}$ & $\rightarrow$ & $\mathrm{CO}$ & $\mathrm{H}_{2} \mathrm{O}$ & & $\checkmark$ & $\checkmark$ & & $G * G^{\prime}{ }_{3}$ \\
\hline 19 & $\mathrm{CH}_{4}$ & $\mathrm{H}_{2} \mathrm{O}$ & $\rightarrow$ & $\mathrm{CO}$ & $3 \mathrm{H}_{2}$ & & $\checkmark$ & $\checkmark$ & $\checkmark$ & $G * G^{\prime}{ }_{5}$ \\
\hline 20 & $\mathrm{C}_{3} \mathrm{H}_{6}$ & $3 \mathrm{H}_{2} \mathrm{O}$ & $\rightarrow$ & $3 \mathrm{CO}$ & $6 \mathrm{H}_{2}$ & & & $\checkmark$ & $\checkmark$ & $G * G_{4}^{\prime}$ \\
\hline 21 & $\mathrm{C}_{3} \mathrm{H}_{8}$ & $3 \mathrm{H}_{2} \mathrm{O}$ & $\rightarrow$ & $3 \mathrm{CO}$ & $7 \mathrm{H}_{2}$ & & $\checkmark$ & $\checkmark$ & $\checkmark$ & $G * G^{\prime}{ }_{5}$ \\
\hline 27 & $14 \mathrm{CeO}_{2} \mathrm{~F}$ & $\mathrm{C}_{3} \mathrm{H}_{8}$ & $\rightarrow$ & $7 \mathrm{Ce}_{2} \mathrm{O}_{3} \mathrm{~F}$ & $3 \mathrm{CO}$ & $4 \mathrm{H}_{2} \mathrm{O}$ & $\checkmark$ & & & $G * G_{2}^{\prime}$ \\
\hline 32 & $14 \mathrm{CeO}_{2} \mathrm{~S}$ & $\mathrm{C}_{3} \mathrm{H}_{8}$ & $\rightarrow$ & $7 \mathrm{Ce}_{2} \mathrm{O}_{3} \mathrm{~S}$ & $3 \mathrm{CO}$ & $4 \mathrm{H}_{2} \mathrm{O}$ & $\checkmark$ & & & $G * G_{2}^{\prime}$ \\
\hline
\end{tabular}

Table S5 Definitions of input gas compositions regarding unmeasurable species in WLTC mode simulations

\begin{tabular}{c|c|c}
\hline \hline \multirow{2}{*}{$\begin{array}{c}\text { Hydrocarbon } \\
\text { species }\end{array}$} & $\mathrm{C}_{3} \mathrm{H}_{6}$ & $45 \%$ of THC measured \\
\cline { 2 - 3 } & $\mathrm{C}_{3} \mathrm{H}_{8}$ & $45 \%$ of THC measured \\
\cline { 2 - 3 } & $\mathrm{CH}_{4}$ & $10 \%$ of THC measured \\
\hline $\mathrm{H}_{2} \quad[\mathrm{~S} 1]$ & {$\left[\mathrm{H}_{2}\right]=\frac{\mathrm{n}}{2} \frac{[\mathrm{CO}]\left([\mathrm{CO}]+\left[\mathrm{CO}_{2}\right]\right)}{[\mathrm{CO}]+\left[\mathrm{CO}_{2}\right] / K_{w}}$} \\
& $\mathrm{~K}_{w}=0.285, n=1.85$ \\
\hline
\end{tabular}

The ratio of $\mathrm{HC}$ among $\mathrm{C} 3 \mathrm{H} 6, \mathrm{C} 3 \mathrm{H} 8$ and $\mathrm{CH} 4$ is quite difficult to detect on a general chassis-dynamo test bench. This time we assumed the ratio to be $0.45: 0.45: 0.10$, respectively, with reference to a relevant work [S2]. 

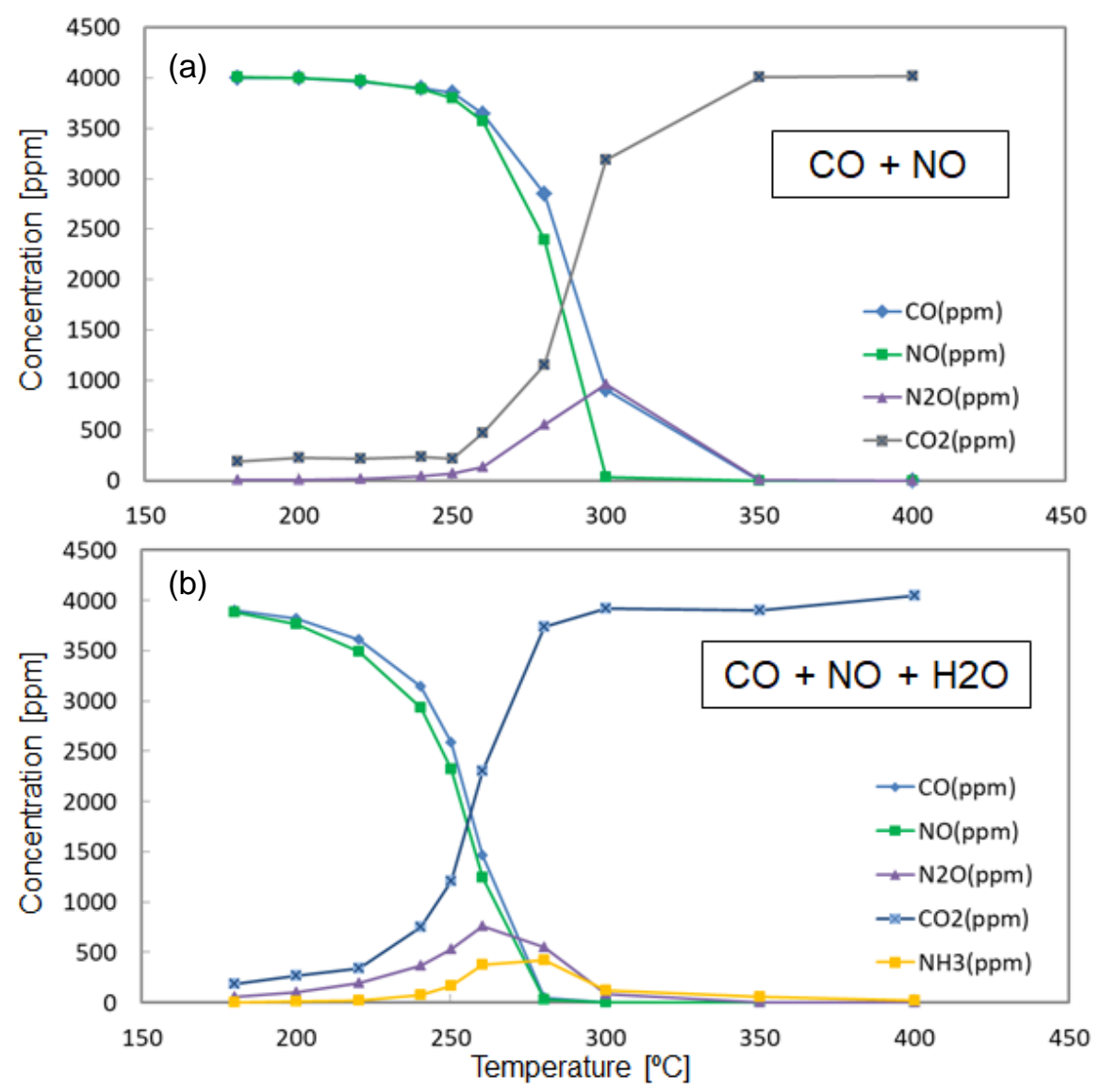

Fig. $\mathrm{S} 1$ Comparisons of TWC out emissions ( $\mathrm{CO}, \mathrm{NO}, \mathrm{N} 2 \mathrm{O}, \mathrm{CO} 2$ and $\mathrm{NH} 3$ ) in $\mathrm{CO}+\mathrm{NO}$ reactions between cases with and without $\mathrm{H}_{2} \mathrm{O}, \mathrm{SV}=82,180 \mathrm{~h}^{-1}$ : (a) $\mathrm{CO}=4,000 \mathrm{ppm}, \mathrm{NO}=4,000 \mathrm{ppm}, \mathrm{N}_{2}$ balance, (b) $\mathrm{CO}=4,000 \mathrm{ppm}$, $\mathrm{NO}=4,000 \mathrm{ppm}, \mathrm{H}_{2} \mathrm{O}=5 \%, \mathrm{~N}_{2}$ balance.
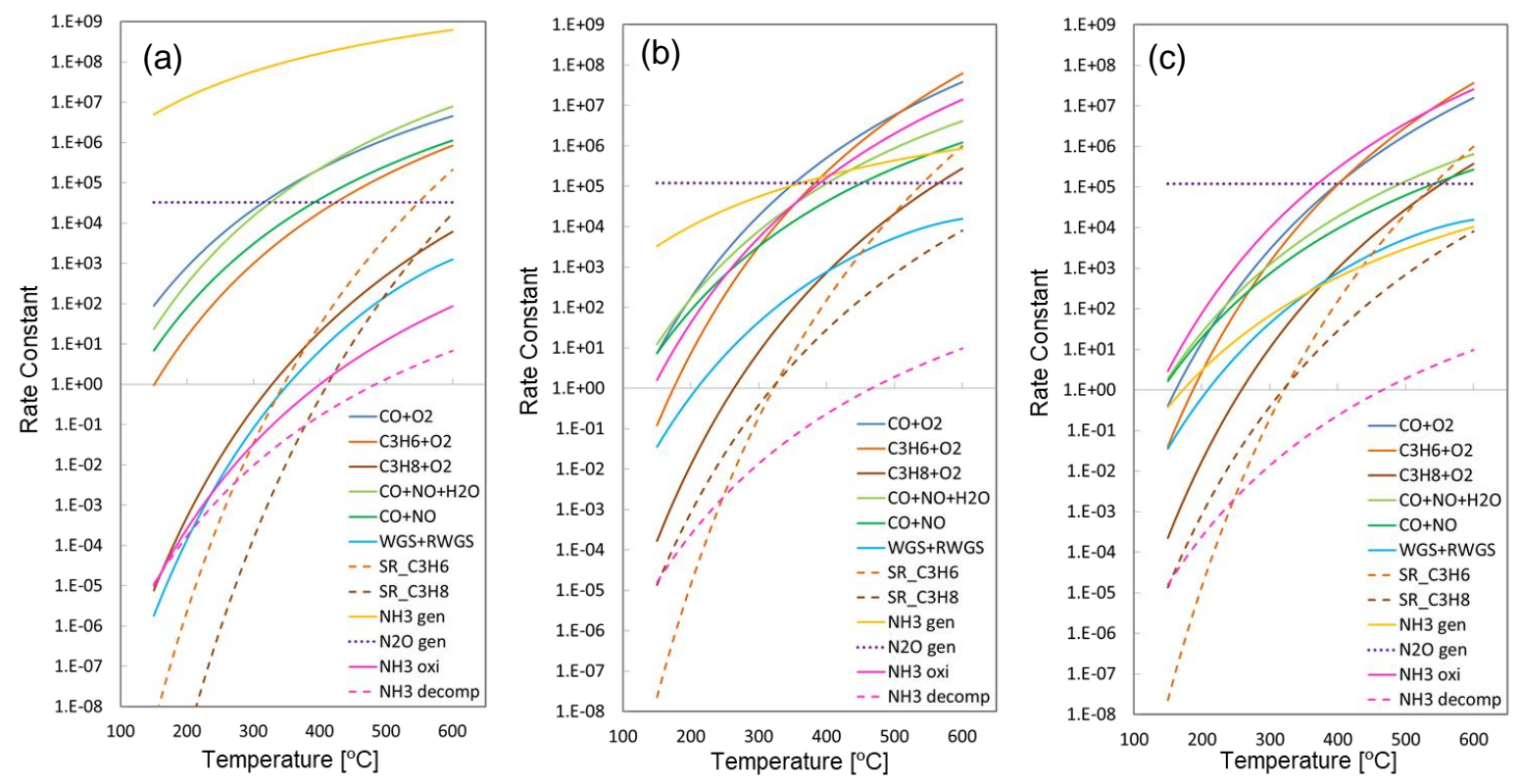

Fig. S2 Comparison of the rate constant as a function of temperature under rich, stoichiometric, and lean conditions: (a) rich; $\lambda=0.91$, (b) stoichiometric; $\lambda=1.00$, and (c) lean; $\lambda=1.03$. 


\section{References}

[S1] Ramanathan, K.; Sharma, C.S. Kinetic Parameters Estimation for Three Way Catalyst Modeling, Ind. Eng. Chem. Res. 2011, 50, 9960-9979.

[S2] Kubo, S. Formation and Emission Characteristics of Unburned Hydrocarbons during Cold Start of a Spark-Ignited Engine System, R\&D Review of Toyota CRDL, 1995, 30, No. 2. 